\title{
JRC2019-1268
}

\section{DEVELOPING FINITE ELEMENT MODELS TO EXAMINE RAIL DEFECTS UNDER COMBINED LOADING}

\author{
Michael Carolan ${ }^{1}$, Benjamin Perlman \\ United States Department of Transportation \\ Volpe National Transportation Systems Center \\ Cambridge, MA, USA
}

\section{ABSTRACT}

One of the Federal Railroad Administration's (FRA's) current areas of research within its rail integrity research program includes investigating the defect growth behavior of modern rail steels. The modern rail steel research is a collaboration among several organizations: ThorntonTomasetti, Arcelor-Mittal, Lehigh University, Harvard University, and the Volpe National Transportation Systems Center (Volpe). A companion paper to this one will describe the results of recently-completed mechanical testing, fracture toughness testing, fatigue crack growth rate calculations, and residual stress field characterizations performed in Phase I of this research.

The behaviors measured in Phase I were examined under laboratory conditions. The effects of the service load environment, including thermal loads, track support conditions, wheel loading, internal defect position and geometry will also need to be investigated for their effects on defect growth. A candidate approach that can be used to investigate these effects is to employ the finite element (FE) method to simulate a variety of conditions. Several of the types of measurements made in Phase I, such as residual stress distribution, serve as inputs to an FE model. Additional inputs, such as the wheel load and support conditions on the rail would be defined based on typical values encountered in the railroad environment. Stress intensity factors can be calculated around each simulated crack front for a given combination of material inputs, load conditions, and defect geometry. These stress intensity factors can then be used to estimate the fatigue crack growth rate under the given conditions.

The modeling approach described above can result in a model that contains several complicated behaviors, including wheel-rail contact, discrete rail supports, and modeling techniques allowing the calculation of stress intensity factors. Further, several of these behaviors require specialized meshing techniques or analysis procedures. Thus, it is essential that the credibility of the model be established through a process of model validation.

This paper lays out a framework for examining individual modeling techniques employed in the model, using a "building block" approach. Rather than trying to assess the entire model of a wheel on a discretely-supported rail containing an internal defect against a test measurement of the same conditions, the model is broken down into several key behaviors that must be verified. These distinct model behaviors, such as the method of discrete support, are then individually compared to known results to develop confidence in the simulation's ability to produce physically-realistic results. In this way, confidence can be developed in the overall, complete model by developing confidence in several of the distinct modeling techniques that are employed in the overall model. The modeling techniques described in this paper include modeling the discretelysupported rail under a wheel load, modeling the internal defect as a crack, and using a submodeling technique to combine areas of coarse and fine mesh in a computationally-efficient manner.

Keywords: Rail steel, defect growth, modeling, simulation, FEA, analysis, verification, validation

$\begin{array}{ll}\text { NOMENCLATURE } \\ \text { BEF } & \text { beam on elastic foundation } \\ \text { FE } & \text { finite element } \\ \mathrm{K}_{\mathrm{I}} & \text { Mode-I stress intensity factor } \\ \text { SIF } & \text { stress intensity factor } \\ \text { V\&V } & \text { Verification and Validation }\end{array}$

${ }^{1}$ Contact author: Michael.Carolan@dot.gov

1

This material is declared a work of the U.S. Government and is not subject to copyright protection in the United States. Approved for public release; distribution is unlimited. 


\section{INTRODUCTION}

One of FRA's areas of research includes investigating the effects of modern rail steel manufacturing techniques on the growth rate of internal defects (such as detail fractures) that can develop within the rail. The modern rail steels characterization project is being conducted by a collaboration that includes Thornton-Tomasetti, Lehigh University, Harvard University, Arcelor Mittal, and Volpe. This paper is written in coordination with a companion paper describing the overall framework of the modern rail steels characterization project, as well as the results obtained during Phase I of this project [1]. In Phase II of this project, FE models are expected to be used to both assist in interpreting the results obtained in Phase I, and to extend the laboratory results obtained in Phase I to service conditions of actual rails in the field.

Figure 1 contains a schematic illustration from the companion paper [1] illustrating the approach previously taken to characterizing defect growth in rails. The right-most column of processes on this figure, outlined with a dashed box, illustrates several of the anticipated uses of modeling in this research program.

The "Fatigue Crack Growth Characterization" process is more fully-explained in the companion paper [1]. One of the desired outcomes of the modern rail steel characterization program is a comparison of the fatigue crack growth rates in service rails manufactured using different processes. The calculation of fatigue crack growth rates depend on input parameters related to material characteristics as well as loading environment. The empirical constants related to material behavior come from the Phase I measurements in this program. The parameters related to load environment, including stress intensity factors $(\mathrm{K})$, must be determined from the anticipated loads that will be acting on the internal defect.

As illustrated in the process labeled "Stress Intensity Factor Calculation” in this figure, the stress intensity factors (SIFs) developing around a particular defect geometry are determined from the residual stresses, the wheel loads, and the thermal stresses acting on the rail. The total stress state (i.e. combined wheel, thermal, and residual stresses) is used to determine the stress intensity factors for a given defect size and position within a rail. The results of a combined wheel-thermal-residual stress analysis are used to calculate stress intensity factors, which are then used as inputs to the "Fatigue Crack Growth Characterization” process.

The "Fatigue Crack Growth Characterization” process has been applied using simplified geometries and assumptions in previous work [2]. To assess the importance of various parameters in the combined stress state, an FE model is one tool that can be used to estimate stress intensity factors under complicated load cases. FE modeling can be used to complement the existing methodology for characterizing fatigue crack growth by analyzing the combined wheel-thermal-residual stress state in a given rail with an internal defect, providing SIF values that are used as inputs to the fatigue crack growth calculations.

An additional use of FE models in the approach outlined in Figure 1 involves developing models to aid in interpreting residual stress results from Phase I. As described in the companion paper to this one, a key result from Phase I was the residual stress state in each type of rail (head-hardened, advanced head-hardened, and standard strength) prior to its entering service. The residual stress state within the rail can have a

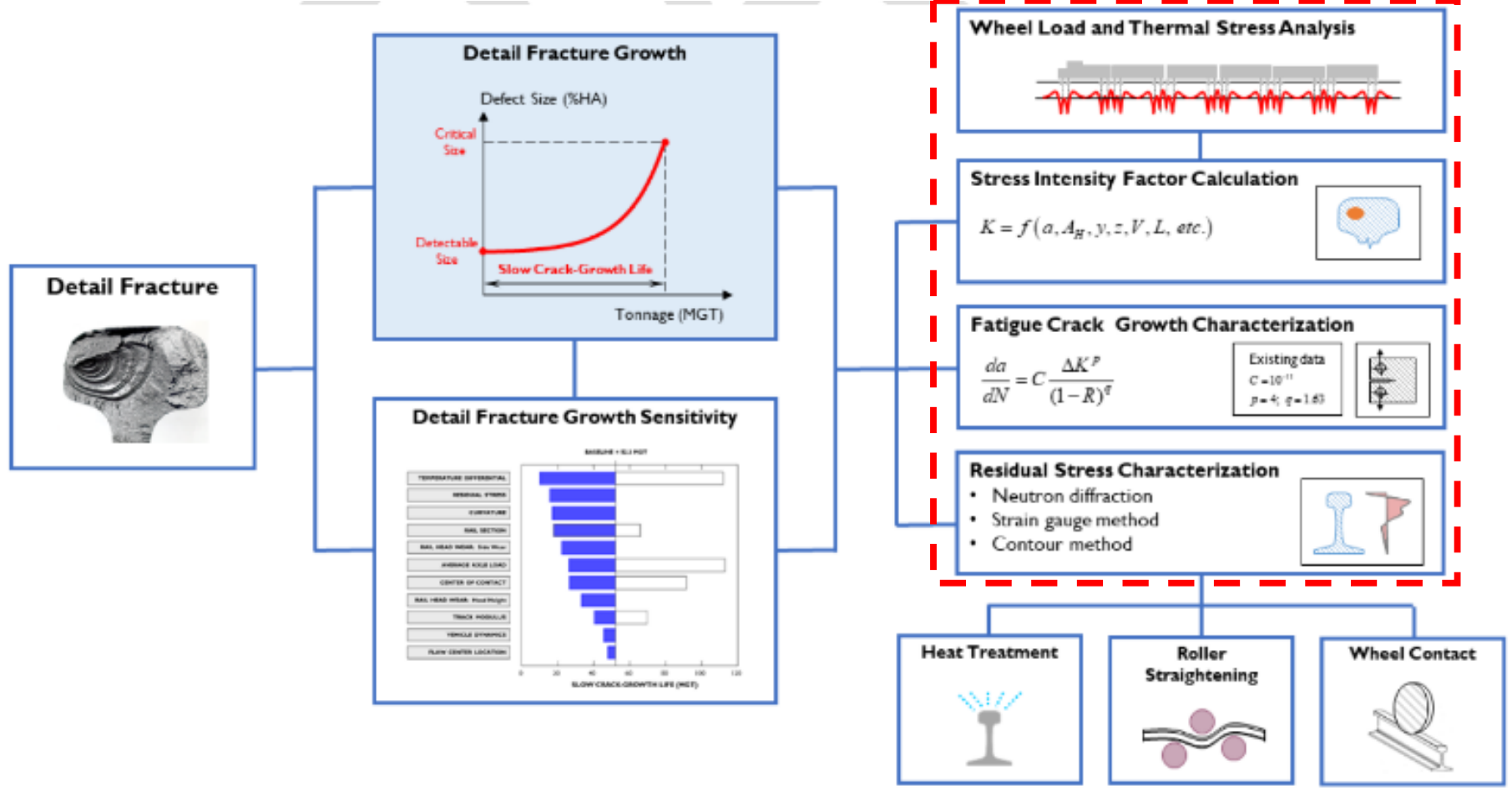

Figure 1. Schematic of Detail Fracture Growth Characterization (from [1])

2

This material is declared a work of the U.S. Government and is not subject to copyright protection in the United States. Approved for public release; distribution is unlimited. 
significant effect on crack growth rates, as a region of the rail may experience tensile stresses without the application of an external load. Any defect that extends into this region of residual tension will experience accelerated growth compared with a defect in an unstressed area, or an area with compressive stresses.

However, since the measurement of residual stresses in both the companion paper and previously-published studies of residual stresses [1, 3] has required cutting the rails, the stress states measured in the cut rails have been altered from the stress states that existed in an uncut rail. Thus, the residual stress states obtained in the laboratory measurements will differ from the residual stress states that would have existed had a sample rail been left intact and placed directly into service. It is expected that FE models can be used to "back-calculate" the stress states in each of the rails that existed prior to the cuts being made. This paper will focus on the modeling techniques that can be used to simulate the load environment; further discussion of the modeling techniques used to reconstruct the residual stress state within the uncut rails is outside of its scope.

\section{APPLICATION OF PHASE I RESULTS TO SERVICE CONDITIONS}

One potential use of FE models as a part of a program investigating crack growth in modern rail steels is to extrapolate beyond laboratory conditions. This type of model would be used to examine the crack growth behavior under load cases that are more representative of the loads to be encountered by a rail installed in track, under "real world" load conditions.

Figure 2 is a schematic illustration of the load environment that is anticipated to be simulated to extend the Phase I results to understand the rail crack growth rates under service conditions. The stresses in a given location within the rail at any point in time are the result of several loading sources. Thermal loads from ambient conditions will either result in tensile or compressive stresses in the rail, depending on the installation conditions. Service loads from passing railcars will place the rail cross-section containing a defect into a combined bending and shearing stress state. This behavior is made more complicated when the effects of multiple wheels acting on the same rail are considered.

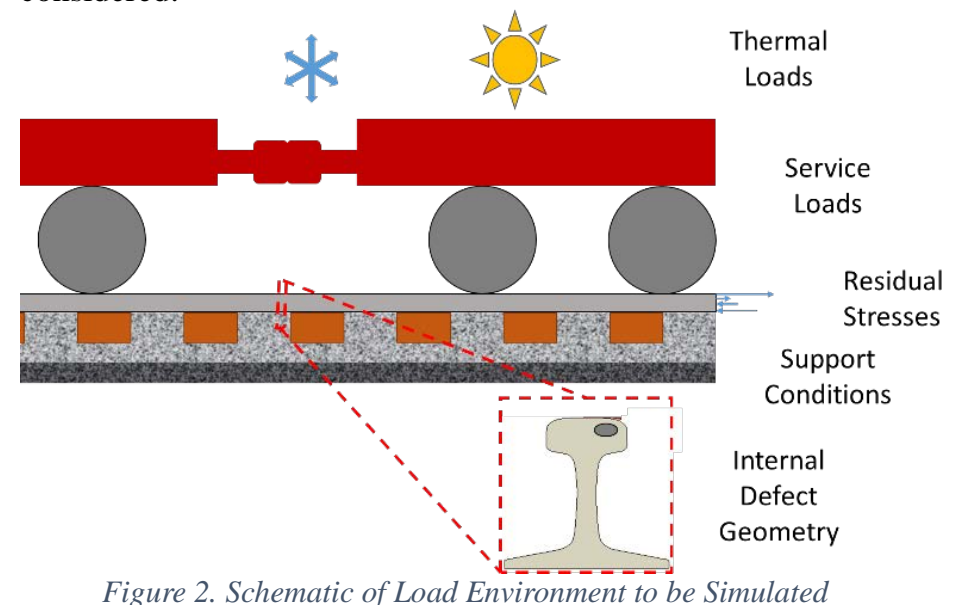

Figure 2. Schematic of Load Environment to be Simulated
The stresses that develop in a particular cross-section of rail containing a defect will be influenced not only by the magnitudes and positions of the wheel loads, but will be influenced by the support conditions provided by the ties, ballast, and subgrade. As shown in Phase I of this program [1] and in previous works [3], the residual stress state within the rail is a spatially-varying 3dimensional stress state. This complicated load environment poses several challenges to being evaluated through testing. Thermal loading conditions will vary with the weather and can be challenging to control over the course of a long-duration outdoor experiment. While service loads can be somewhat controlled, in-service tracks would be expected to see a variety of wheel loads and spacing from different types of vehicles. While support conditions may be initially known and controlled, the support conditions can evolve with increasing traffic over the cross-section of interest. Even if an internal defect can be detected, its exact geometry and position within the rail may not be knowable without cutting the rail open to make the defect visible. Inherent variability within such a test setup poses many challenges to comparing analytical calculations, as conditions a short distance away (e.g. tie support conditions) may be different from those in the area of interest.

In light of this inherent variability, a study may be designed to examine the relative influences of several of the loading parameters previously described. These difficult-to-control parameters of a study of the fatigue crack growth rate under service conditions make this problem an attractive one to be modeled rather than tested. FE modeling offers the ability to control the parameters of the load environment more tightly than could occur in a physical test, and to maintain conditions that would be undesirable to have change during a test.

Modeling introduces its own set of challenges to evaluating the defect growth-rate problem. The complex interactions between environment, vehicle, track, and a rail with an internal defect create many opportunities for a modeling technique or assumption to produce a misleading result. The assumptions and simplifications made in setting up such a model may be necessary or convenient, but it is critically important that those decisions are first examined to determine that they are reasonable.

\subsection{Modeling Framework}

The process of generating evidence and establishing credibility in computer models of physical systems is referred to as verification and validation (V\&V) [4]. The complete model of a railroad rail with an internal defect subjected to differing load environments does not have corresponding experimental data to use in comparisons to help develop confidence in the model through a process of model validation. To develop confidence in this complete model, a series of smaller models have been developed. Each smaller "component" model is meant to examine one behavior for which experimental or closed-form solutions are available. Two such modeling components are described in this paper. These models were executed in the Abaqus/Standard FE software [5]. These component models use the same modeling techniques as are planned to be combined into

3

This material is declared a work of the U.S. Government and is not subject to copyright protection in the United States. Approved for public release; distribution is unlimited. 
the complete model. If each component model, using the same modeling techniques as the complete model, provides reasonable agreement with an independent solution, there is increased confidence in the complete model incorporating multiple such components.

The advanced modeling techniques expected to be used in a complete model include either a continuous or discrete foundation to represent the support provided by the railroad ties and ballast, a simulated flaw within the rail used to calculate stress intensity factors (SIFs) that can then be used to calculate crack growth rates, and submodeling techniques to reduce the runtime necessary to simulate a variety of defect geometries in a reasonable timeframe. This paper presents results of the investigations performed on each of these modeling components to develop confidence in these individual behaviors. Additional $\mathrm{V} \& \mathrm{~V}$ may be necessary and appropriate to understand the influence of further or additional components beyond those presented here.

\section{MODELING OF RAIL SUPPORT CONDITIONS}

The complete model is intended to capture the stresses and deflections of a rail under one or more wheel loads. The rail must be supported in a way that is representative of the way in which an actual rail would be supported. Rail support is provided by the tieplates and crossties, which are themselves supported by a layer of ballast. As a simplification of the support provided by the ballast and subgrade, previous modeling efforts have modeled the ties as a series of discrete springs [6][7][8][9][10]. Previously, bending moments were estimated for discretely versus continuously-supported rail and reported in a technical paper [11]. The work described in this paper also featured discrete springs to represent the rail support provided by the ties, and included discussion of the effect of tie spacing on the validity of the assumption of a continuous, elastic foundation beneath the rail. For sufficiently-close ties, the elastic spring approach provided reasonable agreement with classical beam on elastic foundation (BEF) [12] calculations, but introduces complexity into an already complex model.

A simplified approach to supporting the rail in the FE model is to assume the discrete ties are sufficiently close-spaced and providing sufficiently-uniform support to be approximated as a continuous foundation. This is the same approach as is used in the BEF closed-form calculations, but implemented in the FE model. The Abaqus FE software allows the user to define a linear "elastic foundation" with a user-defined stiffness per area. This modeling approach was investigated as a part of the overall model verification efforts. The stress and deflection responses from the FE model were compared to the stresses and deflections calculated using BEF theory.

To illustrate the comparison, an example problem was evaluated using typical values for rail cross-section, foundation modulus, and wheel load. To simplify the calculations, only a single rigid, cylindrical wheel was modeled in this componentlevel examination. A static load corresponding to one-eighth the weight of a 286,000 pound railcar was applied through this wheel. The parameters common to both the FE models and the BEF calculations are shown in Table 1.

Table 1. Parameters Used in BEF and FE Modeling Verification

\begin{tabular}{|c|c|}
\hline Parameter & Value \\
\hline Rail Section & $136 \mathrm{RE}$ \\
\hline Moment of Inertia & $94.2 \mathrm{in}^{4}$ \\
\hline Foundation Modulus & $100-10,000 \mathrm{psi}$ \\
\hline Wheel Load & 35,750 pounds \\
\hline $\mathrm{E}_{\text {steel }}$ & $3 \times 10^{7} \mathrm{psi}$ \\
\hline
\end{tabular}

The same model used to investigate the rail support conditions can also be used to investigate the influence of length of rail modeled against the global behavior of the model. In general, this preliminary review verified the expected result obtained from BEF theory, that a softer foundation will require a longer length of rail to be included in the model for the BEF-like behaviors to be captured. From BEF theory [12], the wavelength of the deflection line of the beam (in this case, the rail) is a function of the foundation stiffness, the modulus of elasticity of the rail's material, and the area moment of inertia of the rail for bending about a horizontal axis, as described by Equation 1:

$$
\lambda=\sqrt[4]{\frac{\mathrm{k}}{4 \cdot \mathrm{E} \cdot \mathrm{I}_{\mathrm{yy}}}}
$$

From BEF theory, the deflection of the rail from a single wheel load will equal zero at periodic distances from the point of wheel load application. These zero-crossings occur at distances from the point of wheel load of:

$$
\mathrm{d}_{\text {zero }}=\frac{\mathrm{n} \cdot \pi}{4 \cdot \lambda}
$$

where $n=3,7,11 \ldots$ To approximate a finite-length rail as a continuous rail using BEF theory, a rail length sufficient to include the second zero-crossing from the point of wheel load application should be used as a minimum length. Using the values given in Table 1 for stiffness, $E$, and moment of inertia with Equation 1 and an $n$-value of 7 in Equation 2, the resulting minimum-lengths of rail are shown in Figure 3 for several foundation moduli examined.

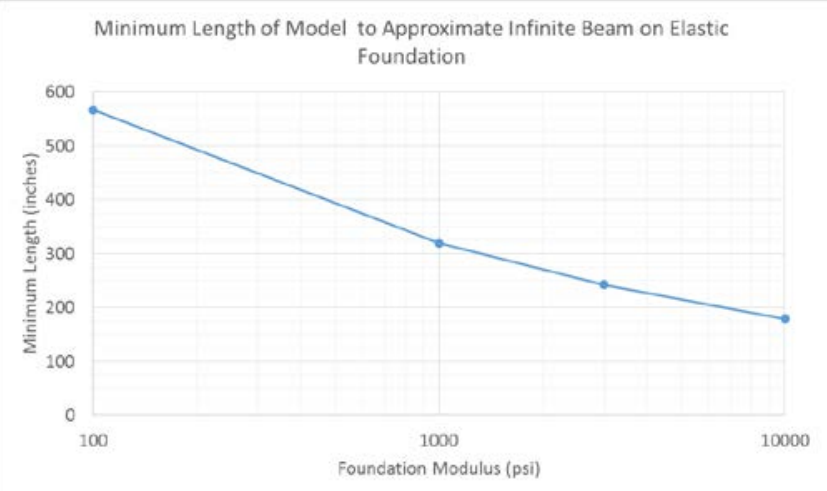

Figure 3. Minimum Length of Model to Approximate Infinite Beam on Elastic Foundation for Various Foundation Moduli

4

This material is declared a work of the U.S. Government and is not subject to copyright protection in the United States. Approved for public release; distribution is unlimited. 
Figure 4 illustrates the rail deflection versus distance along the rail for varied foundation stiffnesses simulated using the FE model. In this figure, the wheel load acts on the rail at a horizontal position of zero inches. For the 100 psi, 1,000 psi, 3,000 psi, and 10,000 psi foundation models, the rail extended beyond the position of the wheel by 250 inches in each direction. The values of extremely soft and extremely strong foundation supports may not represent actual service conditions. However, these values were included in the examination as extreme cases, to illustrate the need to model a sufficiently-long section of rail over the likely range of conditions to be encountered. On the basis of the results from Table 2, this length of rail would be sufficient to capture the second zero-crossing for foundation moduli of 3,000 psi and 10,000 psi, but would not be sufficientlylong for the 1,000 and 100 psi foundation moduli.

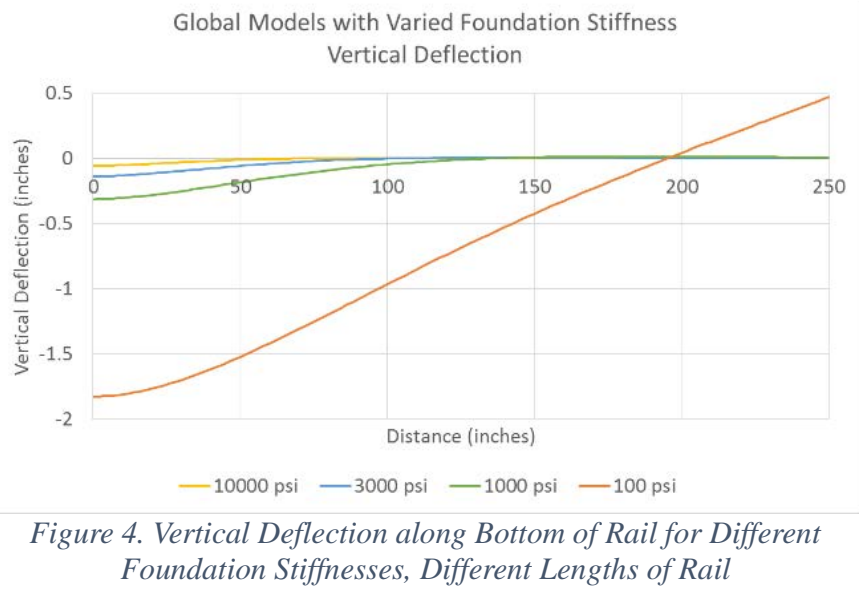

The deflection of a 250 -inch long rail on either a 1,000 or 100 psi foundation did not return to zero as the distance from the wheel increased. While the 1,000 psi results resemble the 3,000 and 10,000 psi results, as the deflection is approaching zero but not reaching it, the 100 psi results are obviously behaving in a very different manner from the other results. This indicated that for this extremely soft foundation, the rail was not behaving as a continuous beam. Further, the 1,000 psi and 100 psi results underscore the need to perform verification checks on the results for reasonable agreement with simplified solutions. In the absence of the results from Figure 3, the 100 psi foundation results would still draw attention to themselves owing to the significant difference in their behavior compared to all the other results. However, the 1,000 psi results bear a much closer resemblance to the results for which 250 inches of rail is a sufficient length.

A foundation stiffness of 3,000 psi was carried forward into subsequent calculations, as this value had been used in previous studies of FE models of rail [6, 10]. A rail length of 250 inches was also carried forward, as a longer rail would require additional simulation time without increasing the fidelity of the model in the region of interest for this foundation stiffness.

The next component-level analysis investigated the use of the "elastic foundation" keyword to model continuous versus discrete support beneath a rail. Two different FE models were created using similar modeling methods. In Model 1, a continuous elastic foundation was uniformly applied to the entire bottom surface of the rail. In Model 2, an elastic foundation was applied to discrete locations, simulating the support provided by regularly-spaced crossties. Rather than applying a uniform elastic foundation to the entire rail base, an effective stiffness was calculated based on the proportion of the length of a rail in contact with crossties over a fixed length of track. This effective foundation was then only enabled under the rail at the locations of cross ties, leaving an unsupported span of rail between areas of foundation. These two approaches are shown schematically in Figure 5, where a wheel is shown on top of Model 1 in the top figure and a wheel is shown on top of Model 2 (loading the rail between discrete foundations) in the bottom of this figure.

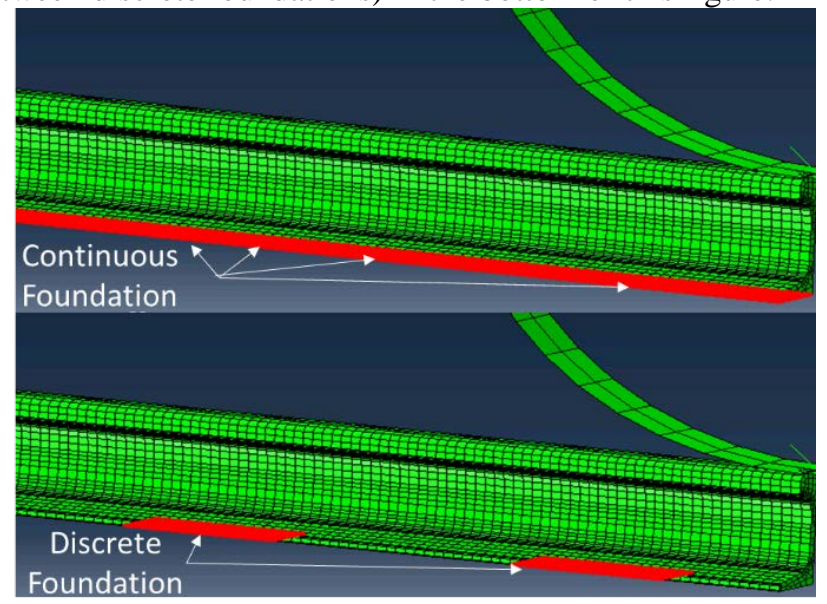

Figure 5. Wheel on Continuous Elastic Foundation (top) and Discrete Elastic Foundation (bottom)

Two of the key results compared between the FE models using discrete and continuous support and the BEF calculations are the axial stresses in the rail base and the vertical deflection of the rail. Figure 6 contains a plot of the axial stress in the bottom of the rail for the two $\mathrm{FE}$ results and the BEF calculations. The discrete and continuous FE results nearly overlay one another, while there is a difference between the FE results and the BEF calculations in the vicinity of the point of load application. For the discrete support case, the load is applied to an unsupported area between two areas of support.

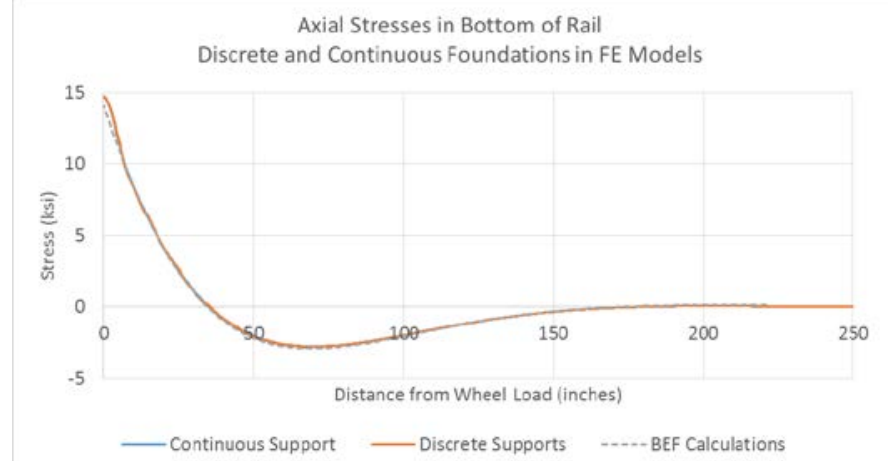

Figure 6. Axial Stresses in Bottom of Rail versus Distance from Wheel Load for FE and BEF Calculations

5

This material is declared a work of the U.S. Government and is not subject to copyright protection in the United States. Approved for public release; distribution is unlimited. 
Additionally, the vertical deflection of the rail as a function of distance from the load was compared both between FE modeling approaches and against the BEF calculations. These results exhibited a similar qualitative level of agreement as the stress results, with a difference in the vicinity of the load application. The peak stresses in the bottom of the rail and the maximum downward deflection results are summarized for the FE analyses and the BEF calculations in Table 2. This table indicates that for all results compared, the FE results exhibit a difference of less than $5 \%$ of the BEF results.

Table 2. Summary of Results, FE and BEF Calculations

\begin{tabular}{|c|c|c|c|c|c|c|c|}
\hline \multicolumn{2}{|c|}{} & $\begin{array}{c}\text { MAXIMUM } \\
\text { DOWNWARD } \\
\text { DEFLECTION }\end{array}$ & $\begin{array}{c}\text { MAX } \\
\text { STRESS } \\
\text { (BOTTOM) }\end{array}$ & $\begin{array}{c}\text { MIN } \\
\text { STRESS } \\
\text { (BOTTOM) }\end{array}$ & $\begin{array}{c}\text { DEFLECTION } \\
\text { DIFFERENCE }\end{array}$ & $\begin{array}{c}\text { MAX STRESS } \\
\text { (BOTTOM) } \\
\text { DIFFERENCE }\end{array}$ & $\begin{array}{c}\text { MIN STRESS } \\
\text { (BOTTOM) } \\
\text { DIFFERENCE }\end{array}$ \\
\cline { 2 - 9 } & in & KSI & KSI & $\%$ & $\%$ & $\%$ \\
\hline $\begin{array}{c}\text { BEF } \\
\text { CALCULATIONS }\end{array}$ & BEF & -0.135 & 14.1 & -2.9 & - & & \\
\hline $\begin{array}{c}\text { CONTINUOUSLY } \\
\text { SUPPORTED FEA }\end{array}$ & PROFILE 1 & -0.139 & 14.7 & -2.8 & $2.7 \%$ & $4.4 \%$ & $-4.3 \%$ \\
\hline $\begin{array}{c}\text { DISCRETELY } \\
\text { SUPPORTED FEA }\end{array}$ & PROFILE 1 & -0.139 & 14.7 & -2.8 & $3.0 \%$ & $4.5 \%$ & $-3.5 \%$ \\
\hline
\end{tabular}

For well-supported track, the discrete and continuous supports do not show much of a difference compared to the BEF calculations. However, for other track conditions (e.g. missing ties, irregularly-spaced ties, varying ballast conditions), the discrete-support model offers increased flexibility to represent spatial variations in track support conditions. While it is more complicated to set up a model to use a discrete support condition, this approach offers flexibility to vary the stiffness of each tie and to vary the spacing between ties.

\section{CRACK MODELING}

In the complete model, the internal defect may be simulated as a circular or elliptical crack in the head of the rail. As a verification of the methods chosen to model the defect and calculate SIFs, a closed-form solution for calculating the stress intensity factor $\mathrm{K}_{\mathrm{I}}$ for a circular crack within a circular bar will be compared with a simulation of the same case [13]. Techniques studied using this particular component verification model included the use of symmetry planes at the crack location, the mesh size necessary to model the crack, and element types used to model the crack. This verification model used the same contour integral calculation techniques to calculate the SIFs as had been previously-employed to examine SIFs associated with a rail base crack using FEA [14].

Figure 7 contains a schematic illustration of the verification case chosen. This case features a round bar with a circular internal defect (crack) subject to uniform tension. Because the crack is coaxial with the circular bar, this problem is axisymmetric. Thus, the SIF is the same around the entire circumference of the circular defect.

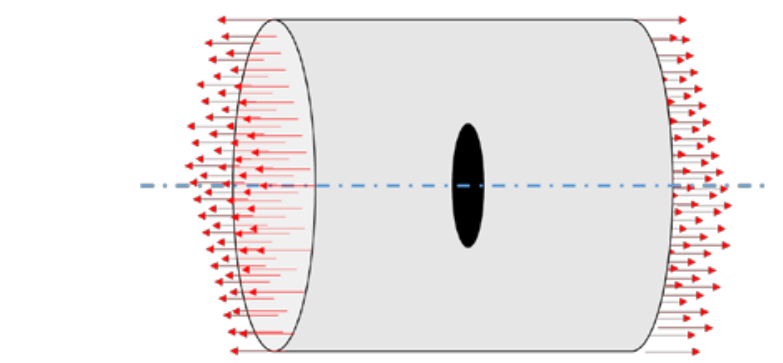

Figure 7. Schematic of Round Bar with Circular Internal Crack under Uniform Tension

The $\mathrm{K}_{\mathrm{I}}$ SIF was calculated using the closed-form approach from Reference 13 for several different combinations of bar diameter and crack diameter. For all models, a uniform pressure load totaling 1,000 pounds of tension was applied to the ends of the bar. The bar was modeled as an elastic steel with a Young's Modulus of $3 \times 10^{7} \mathrm{psi}$. The bar was modeled as either a 4- or 5inch long bar of circular cross-section. The length of bar beyond the crack is important to ensure that a uniform stress field can develop in the cross-section far from the crack.

Developing an axisymmetric model would be an appropriate simplification for the round bar with a circular crack. However, the purpose for performing this component-level examination is to develop confidence in modeling techniques that will be applied to the complete model of an internal defect within a rail in a service environment. Because a detail fracture within the head of a rail is not an axisymmetric situation, it would not be appropriate to employ an axisymmetric model at the component verification level. However, depending on the position of the detail fracture within the rail and the relative position of the wheels within the complete model, one or more planes of symmetry may exist within that model. Thus, while it is not appropriate to perform component-level simulations on the round bar model using axisymmetric conditions, it is appropriate to consider using different combinations of planar symmetry simplifications at the component level.

This approach can examine whether the selected implementation of $\mathrm{K}_{\mathrm{I}}$ calculations within the Abaqus solver provides consistent results when symmetry conditions are employed to reduce the model size. This is an important check to include, as this modeling approach involves a path integral around the crack front. If a symmetry plane passes through the crack front itself, it is important to verify that the calculations are taking this symmetry into account.

The chosen method of modeling a crack within a solid body uses a user-defined number of contours surrounding the crack tip to calculate SIFs. Because the contour method is, in theory, path independent, evaluating the SIFs using multiple paths around the crack front can provide an indication of mesh convergence. That is, if results using $n$ different contour around the same crack are found to give SIFs that are very close to one another, this indicates the mesh is converging and the SIF calculations really are mesh independent. However, if there is disagreement among the contours, it is an indication that further mesh refinement may 
Table 3. Summary of Round Bar with Round Crack Verification Models

\begin{tabular}{|c|c|c|c|c|c|c|c|c|c|c|}
\hline Model & $\begin{array}{c}\text { Bar } \\
\text { Radius }\end{array}$ & $\begin{array}{c}\text { Crack } \\
\text { Radius }\end{array}$ & Crack Location & \multicolumn{3}{|c|}{ Symmetry Used } & $\begin{array}{c}\text { Axial Length } \\
\text { (Overall) }\end{array}$ & $\begin{array}{c}\text { Axial Length } \\
\text { (Crack Zone) }\end{array}$ & $\begin{array}{c}\text { Element } \\
\text { Type }\end{array}$ & $\begin{array}{c}\text { Number of } \\
\text { Elements }\end{array}$ \\
\hline- & in. & in. & - & Width & Height & Length & in. & in. & - & - \\
\hline \hline Bar-1 & 5 & 1 & Center & - & - & Half & 4 & 0.2 & C3D20 & 84,580 \\
\hline Bar-2 & 5 & 1 & Center & Half & Half & - & 4 & 0.2 & C3D20 & 47,256 \\
\hline Bar-3 & 5 & 1 & Center & Half & Half & Half & 5 & 0.2 & C3D20R & 43,980 \\
\hline Bar-4 & 5 & 1 & Center & Half & Half & Half & 5 & 0.2 & C3D20 & 43,980 \\
\hline Bar-5 & 4 & 0.5 & Center & Half & Half & Half & 5 & 0.1 & C3D20 & 45,906 \\
\hline Bar-6 & 4 & 1 & Center & Half & Half & Half & 5 & 0.2 & C3D20 & 51,738 \\
\hline Bar-7 & 4 & 1 & 1" Lateral Offset & - & Half & Half & 4 & 0.2 & C3D20 \\
\hline
\end{tabular}

be necessary.

Figure 8 shows an exemplar FE mesh from a model using three planes of symmetry to reduce the model to one-eighth its complete size. All FE models used in this component-level investigation were meshed using similar, but not identical, techniques. In all cases, the model used 20-node (quadratic) hexahedral (brick) elements. For the elements adjacent to the crack tip, the mid-side node was moved to the quarter-point of the element's face to provide a more accurate solution. From leftto-right, this figure shows the full domain of the model, a zoomed-in view of the mesh around the circular crack, and a further zoomed-in view of the mesh around the crack tip. The roughly concentric arrangement of elements surrounding the crack tip is deliberately chosen to facilitate the contour method of calculating $\mathrm{K}_{\mathrm{I}}$.

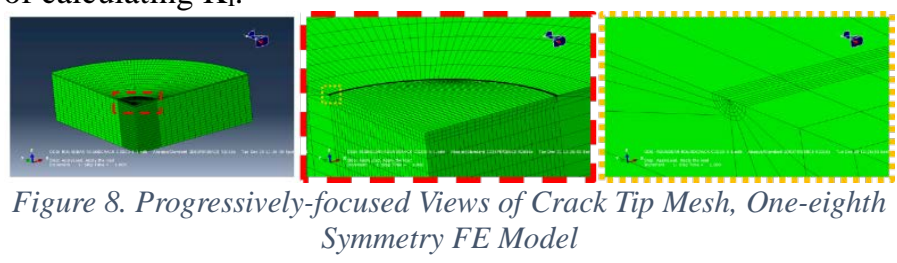

Seven FE models of the round bar problem were run. Each model featured a unique combination of bar radius, crack radius, symmetry conditions, axial length, and element type. As a result of these variations, the total number of elements also varied from model to model. Both the closed-form solution and the FE models featured a coaxial circular defect within a round bar, with the exception of one simulation that featured a circular defect offset from the center of the round bar. This case was included in the component-level verification to examine the effects of including an asymmetric case, as the complete model is expected to be used to evaluate load cases where the rail defect is loaded asymmetrically. The seven FE models are summarized in Table 3.

Due to space limitations, a full discussion of the results of each of the seven bar models is not possible within this paper. Several key results are instead presented below, with an accompanying discussion describing the lessons that were learned from this component-level examination. Table 4 contains a summary of results for each closed-form solution and FE models Bar-1 through Bar-6. Results from model Bar-7 are not included in this comparison, as this model featured an offset defect in the bar, for which there is not a corresponding closedform solution. Three different closed-form solution results are presented, as each combination of bar radius and crack radius results in a different value for $\mathrm{K}_{\mathrm{I}}$. Since each $\mathrm{FE}$ model exhibited a variation in $\mathrm{K}_{\mathrm{I}}$ values around the circumference of the circular crack, results are presented as the minimum and maximum values obtained from each model.

Table 4. Summary of KI Values and Differences between FE Models and Closed-form Solutions

\begin{tabular}{|c||c|c|c|c|}
\hline \multirow{2}{*}{ Analysis } & \multicolumn{2}{|c|}{$\mathbf{K}_{\mathbf{I}}$} & \multicolumn{2}{c|}{ Difference from Closed-form } \\
\cline { 2 - 5 } & \multicolumn{2}{|c|}{ PSI-IN ${ }^{1 / 2}$} & \multicolumn{2}{c|}{$\%$} \\
\cline { 2 - 5 } & Min. & Max. & Min. & Max. \\
\hline Closed-form 5"/1" & 14.4 & 14.4 & - & - \\
\hline Bar-1 & 14.5 & 14.7 & $0.5 \%$ & $2.1 \%$ \\
\hline Bar-2 & 14.3 & 14.6 & $-1.1 \%$ & $1.0 \%$ \\
\hline Bar-3 & 18.4 & 18.4 & $27.3 \%$ & $27.7 \%$ \\
\hline Bar-4 & 14.0 & 14.3 & $-2.7 \%$ & $-1.3 \%$ \\
\hline Closed-form 4"/0.5” & 15.9 & 15.9 & - & - \\
\hline Bar-5 & 15.3 & 16.0 & $-3.6 \%$ & $0.4 \%$ \\
\hline Closed-form 4"/1" & 22.7 & 22.7 & - & - \\
\hline Bar-6 & 21.5 & 22.0 & $-5.0 \%$ & $-2.8 \%$ \\
\hline
\end{tabular}

From these results, one apparent standout result is the considerably higher differences between the closed-form solution and model Bar-3. One variable examined at the component level was the use of fully-integrated (C3D20) or reduced-integration (C3D20R) elements in the Abaqus software to mesh the region around the crack. Models Bar-3 and Bar-4 were identical, except for the element formulation; Bar-3 used reduced-integration elements, and Bar-4 used fully-integrated elements. In model Bar-3, the reduced-integration results consistently over-predict the $\mathrm{K}_{\mathrm{I}}$ value by approximately $27 \%$. In model Bar-4, the fully-integrated element results are much closer to the closed-form solution. It is clear that the fully-integrated 7

This material is declared a work of the U.S. Government and is not subject to copyright protection in the United States. Approved for public release; distribution is unlimited. 
elements provide a superior level of agreement compared to the reduced-integration elements for the same level of mesh refinement. Thus, fully-integrated quadratic (C3D20) elements will be carried forward for modeling cracks in the complete model.

\section{SUBMODELING TECHNIQUE}

Based on the component-level study performed on modeling of the rail support conditions, it is apparent that for typical foundation stiffnesses of 1,000 psi or greater, the modeling domain must extend at least 250 inches beyond the position of the wheel to correctly capture the behavior of the rail beneath the wheel. If multiple wheels are included within the model the modeling domain would extend at least 250 inches beyond the farthest wheel from the rail cross-section of interest to ensure that its deflection field was correctly captured by the model. While symmetric boundary conditions can provide some computational efficiency, the modeling domain would still be quite large compared to the diameter of a single wheel.

The crack modeling study has shown that the contour approach to calculating SIFs requires a highly-refined mesh of higher-order (quadratic) fully-integrated elements. This refined mesh is highly-localized around a defect that measures only a few inches in each direction. These elements are necessary to calculate the $\mathrm{K}_{\mathrm{I}}$ values while minimizing the error from the closed-form solution, but increase computational time due to the additional mid-side nodes and integration points within the elements.

The combination of a large modeling domain necessary to capture the global behavior with a highly-refined, higher-order mesh necessary to capture the localized stresses around the internal defect has the potential to be computationally demanding. While the mesh can be graded to use coarser elements farther away from the cross-section containing the defect, the elements are limited in how extreme an aspect ratio they can have and still produce credible results.

A potentially more efficient approach to addressing the competing mesh requirements is to use a submodeling technique. With this approach, a global model of the complete rail, without a defect, is used to simulate the desired loading scenario. Because the crack is a local effect, the global reaction forces, farfield stresses, and global deflections should experience an insignificant influence from the presence of a defect within the rail. A small model of rail section incorporating the desired defect, referred to as a submodel, is also created. This model would feature the refined, higher-order mesh necessary to capture the stress intensity factors surrounding the internal crack. The stresses and deflections obtained from the global model are then projected onto the limits of the submodel. If the submodel is sufficiently-large to place the defect away from its boundaries, the far-field stresses and deflections from the global model are unaffected by the presence of the defect. Ideally, this approach to modeling produces the same result as would have been obtained by running a highly-detailed global model with an internal defect, but does so in less time.
One of the fundamental assumptions that must be true for this approach to be effective is that the presence of the defect within the submodel has only local effects, as the global model is run without any sort of defect. Additionally, while in theory the global model and the submodel can use elements of a different size and different order, care must be taken to ensure that the projection of the results from the global model into the submodel are reasonable and are capturing the behavior(s) of interest. While a fine mesh of higher-order elements is required in the region surrounding the crack, submodeling can theoretically allow a coarse mesh of simple elements to model the global rail. However, it is important to verify that the submodeling constraints can effectively map the global model displacements or stresses onto the boundaries of the submodel when the two models have different element sizes and orders.

Investigation of the submodeling approach was done using a simplified model of a rail segment on a continuous elastic foundation with an equivalent modulus of 3,000 psi, subjected to a single wheel load. The global model consisted of an 800-inch segment of rail with a coarse mesh. Other model parameters were the same as previously reported in Table 1 . The submodel consisted of a 4-inch length of rail directly under the wheel. The submodel was meshed using a much finer mesh than the global model. The geometry (left) and meshes (right) of both the global model (top) and submodel (bottom) are shown in Figure 11. Because of symmetry, both the global model and the submodel used quarter-symmetric representations of the rail.

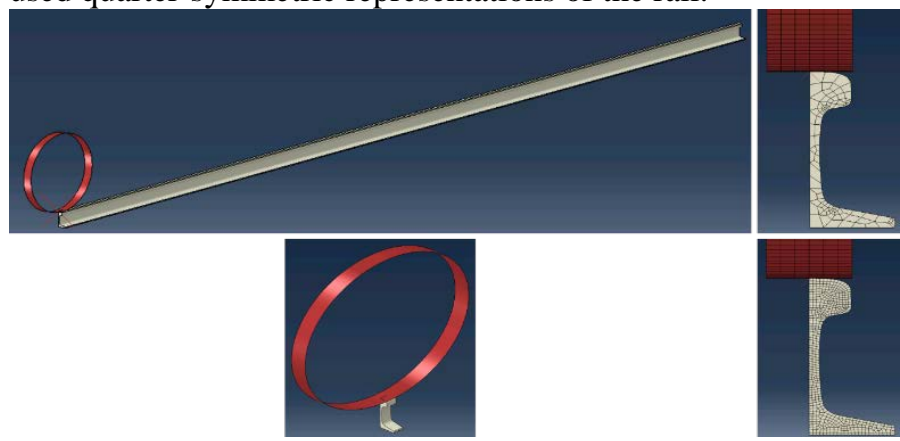

Figure 9. Model Overview (left) and Cross-section Mesh (right) for Global Model (top) and Submodel (bottom)

For both the global model and the submodel, the same meshes were used in the various simulations performed in this component-level verification. However, different element formulations were used in the models to evaluate how well the submodeling technique worked for different combinations of elements in the global model and submodel. The Abaqus software requires the user to define a position tolerance between the nodes involved in the submodel constraint on the submodel and on the global model to account for differences in mesh density between the two models. In general, a value between 0.1 inches and 0.25 inches was used.

As the ultimate goal of this modeling effort is to develop a model of a rail which features an internal defect under service conditions, it was important to consider the results of the crack modeling previously discussed (see Crack Modeling). Because

\section{8}

This material is declared a work of the U.S. Government and is not subject to copyright protection in the United States. Approved for public release; distribution is unlimited. 
the crack modeling approach used in this effort required higherorder elements in the crack region, the submodel should only be meshed using higher-order elements. While the crack modeling verification study demonstrated that fully-integrated higherorder elements produced better agreement with published solutions for $\mathrm{K}_{\mathrm{I}}$, fully-integrated elements are only needed in the mesh immediately surrounding the crack. Reduced integration higher-order elements may be used in the same mesh as fullyintegrated higher-order elements without the need to write constraint equations. Additionally, first-order reduced (C3D8R) and full (C3D8) integration elements were investigated in the global model.

When evaluating each combination of global model and submodel meshes, two different comparisons have been made. First, the stresses and displacements of the global model are compared to the stresses and displacements calculated using BEF theory. This comparison provides a relative indication of the performance of different element orders and formulations for the global model. Second, the stresses and deflections along the length of the submodel are compared with the stresses and deflections in the corresponding locations in the global model. Because this exercise is being conducted without including a defect within the submodel, the stresses and deflections in the submodel should be the same as those in the corresponding locations within the global model. Any discrepancies between the two results would indicate that the submodeling constraint is not functioning as intended for that combination of global and submodel. The stress results from the bottom of the rail at a distance 2" down the rail from the wheel (the end of the submodeled domain) are presented in Table 5 for the BEF calculations, and each combination of global model and submodel investigated.

Table 5. Summary of Global and Submodeling Results

\begin{tabular}{|c|c|c|c|c|c|}
\hline & \multirow[t]{2}{*}{ Analysis } & $\begin{array}{c}\text { Element } \\
\text { Type }\end{array}$ & $\begin{array}{c}\text { Stress 2" } \\
\text { from } \\
\text { Wheel } \\
\text { (Bottom) }\end{array}$ & $\begin{array}{l}\text { Difference } \\
\text { from BEF }\end{array}$ & $\begin{array}{c}\text { Difference } \\
\text { from } \\
\text { Global } \\
\text { Model }\end{array}$ \\
\hline & & - & KSI & $\%$ & $\%$ \\
\hline & BEF & - & 12.8 & - & - \\
\hline \multirow{10}{*}{ 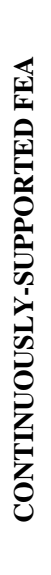 } & GLOBAL & C3D8R & 12.3 & $-4.2 \%$ & - \\
\hline & SUBMODEL & C3D20R & 13.6 & $6.4 \%$ & $11.1 \%$ \\
\hline & GLOBAL & C3D8 & 12.9 & $1.1 \%$ & - \\
\hline & SUBMODEL & C3D20R & 13.4 & $4.5 \%$ & $3.4 \%$ \\
\hline & GLOBAL & C3D20R & 13.9 & $8.4 \%$ & - \\
\hline & SUBMODEL & C3D20R & 13.9 & $8.5 \%$ & $0.1 \%$ \\
\hline & GLOBAL & C3D20 & 13.9 & $8.8 \%$ & - \\
\hline & SUBMODEL & C3D20 & 14.0 & $9.0 \%$ & $0.2 \%$ \\
\hline & GLOBAL & $\begin{array}{l}\text { C3D8R/ } \\
\text { C3D20R }\end{array}$ & 13.9 & $8.5 \%$ & - \\
\hline & SUBMODEL & C3D20R & 14.0 & $9.1 \%$ & $0.6 \%$ \\
\hline
\end{tabular}

The results shown in Table 5 appear to indicate a tradeoff between the ability of the global model to agree with the BEF stress results and the ability of the submodel to agree with the global model at the same location. The two highlighted cells indicate the smallest differences between the global model and the BEF result or the smallest difference between the global model and the submodel. For the result with the smallest difference between the global result and the BEF result, the difference between the submodel and global model is the secondlargest of all seen. For the case with the smallest difference between the submodel and global model, each of those models exhibits a fairly large difference when compared with the BEF result.

Further examination may be appropriate to investigate additional combinations of global and submodeling meshes, or whether a global reduction in mesh size can improve the level of agreement for both behaviors. A desired outcome would be a combination of local and global meshes for which the differences between the FE models and the BEF calculations are small, and simultaneously the difference between the global model and submodel at the limits of the submodel domain is also small.

\section{CONCLUDING REMARKS}

One of the areas of research currently pursued by the FRA is investigating the effects of modern rail steel manufacturing techniques on the growth rate of internal defects that can develop within the rail. Phase I of this project included laboratory characterizations on the steels, including mechanical and chemical analyses, and residual stress characterization. In Phase II of this project, FE models are expected to be used to both assist in interpreting the results obtained in Phase I, and to extend the laboratory results obtained in Phase I to service conditions of actual rails in the field.

An FE model simulating the service load environment of a rail containing an internal defect is anticipated to contain several complicated behaviors and require advanced modeling techniques to capture these behaviors. As the model grows in complexity, it is essential that confidence be developed in the obtained by the model, as test data will not exist for comparison with the model. This paper described an approach for developing confidence in the model where the complete model of a rail under service conditions is broken into several smaller "component" models, each of which is intended to evaluate a particular modeling technique. By developing confidence in individual modeling components, confidence can also be developed in the complete model if those same techniques are carried forward into that model. The components examined in this paper included the length of rail necessary to capture the response of a single wheel load, the use of an intermittent or continuous foundation beneath the rail, the use of a contour integral method to estimate $\mathrm{K}_{\mathrm{I}}$, and the use of submodeling to improve computational efficiency. Additional V\&V activities may be appropriate to include in future studies if additional details, such as wheel-rail contact stresses, are anticipated for inclusion in an analysis performed using the complete model.

\section{9}

This material is declared a work of the U.S. Government and is not subject to copyright protection in the United States. Approved for public release; distribution is unlimited. 


\section{ACKNOWLEDGEMENTS}

The work described in this paper was performed as part of the Rail Integrity task within the Track Systems Research Program of the FRA Office of Research, Development, and Technology. The authors appreciate the support and guidance provided by Dr. Robert Wilson, Program Manager, Office of Railroad Policy and Development.

The work in the Modern Rail Steels characterization project was performed by Pawel Woelke (Thornton-Tomasetti), Herman F. Nied, John N. Dupont, Sena Kizildemir (Lehigh University), John W. Hutchinson (Harvard University), and Fred B. Fletcher (Arcelor Mittal).

The authors wish to acknowledge the invaluable technical contributions of Dr. David Jeong to the rail integrity research program.

\section{REFERENCES}

[1] Jeong, D.Y., Woelke, P.B., Nied, H.F., DuPont, J.N., Kizildemir, S., Hutchinson, J.W., and Fletcher, F. B. Defect Growth Characterization in Modern Rail Steels. Proceedings of the 2019 ASME Joint Rail Conference, JRC2019-1265, Snowbird, UT, April 2019.

[2] Lyons, M.L., Jeong, D.Y., Gordon, J.E., Fracture Mechanics Approach to Estimate Rail Wear Limits, Proceedings of the 2009 ASME Rail Transportation Fall Technical Conference, RTDF2009-18035, Fort Worth, Texas, October 2009. http://ntl.bts.gov/lib/35000/35200/35228/rtdf2009-18035.pdf

[3] Luzin, V., Prask, H.-J., Gnaupel-Herold, T., Gordon, J., Wexler, D., Rathod, Ch., Pal, S., Daniel, W., and Atrens, A. (2013). Neutron Residual Stress Measurements in Rails; Scientific Reviews; Vol. 24; No. 3.

[4] ASME V\&V 10-2006. Guide for Verification and Validation in Computational Solid Mechanics. Issued December 29, 2006.

[5] Dassault Systèmes. Abaqus/Standard R2016.

[6] Carolan, M.E., Jeong, D.Y., Perlman, A.B., Engineering Studies on Joint Bar Integrity, Part II: Finite Element Analysis, Proceedings of the 2014 Joint Rail Conference, JRC2014-3708, April 2014. http://ntl.bts.gov/lib/51000/51500/51593/JRC2014Joint_bar_integrity.pdf

[7] Zhu, K., Edwards, J. R., Qian, Y., \& Andrawes, B. (2016). Finite element analysis of the effects of bolt condition on bolted rail joint stresses. Transportation Research Record, 2545, 36-45. https://doi.org/10.3141/2545-05

[8] Zhu, K., Qian, Y., Riley Edwards, J., \& Andrawes, B. O. (2017). Finite Element Analysis of Rail-End Bolt Hole and Fillet Stress on Bolted Rail Joints. Transportation Research Record, 2607(1), 33-42. https://doi.org/10.3141/2607-06
[9] Yin, H., Qian, Y., Riley Edwards, J., \& Zhu, K. (2018). Investigation of Relationship between Train Speed and Bolted Rail Joint Fatigue Life using Finite Element Analysis. Transportation Research Record. https://doi.org/10.1177/0361198118784382

[10] Talamini, B., Jeong, D.Y., and Gordon, J., Estimation of the Fatigue Life of Railroad Joint Bars, ASME/IEEE Joint Rail Conference \& Internal Combustion Engine Spring Technical Conference, JRCICE2007-40065, Pueblo, CO, March 2007. https://rosap.ntl.bts.gov/view/dot/8986

[11] Jeong, D.Y. and Perlman, A.B. (2011) Estimating Track Capacity Based on Rail Stresses and Metal Fatigue. RTDF2011-67001. Proceedings of the ASME 2011 Rail Transportation Division Fall Technical Conference. https://rosap.ntl.bts.gov/view/dot/9481

[12] Hetényi, M., Beams on Elastic Foundation, University of Michigan Press, Ann Arbor, MI, 1974.

[13] H. Tada, P. Paris, and G. Irwin, The Stress Analysis of Cracks Handbook, Del Research Corporation, 1973.

[14] Jeong, D.Y., Carolan, M.E., Yu, H., Perlman, A.B., Fracture Mechanics and Beam Theory Analyses of SemiElliptical Cracks Originating in the Base of Rail, Proceedings of the 2012 ASME/ASCE/IEEE Joint Rail Conference, JRC201274027, Philadelphia, Pennsylvania, April 2012. http://ntl.bts.gov/lib/44000/44600/44615/Jeong_Fracturemecha nicsBeam.pdf

\section{APPENDIX A - FOUNDATION MODELING DETAILS}

To implement an equivalent 3,000 psi trackbed stiffness in the Abaqus FE software using the "Elastic Foundation" approach, the model requires that the desired stiffness value be divided by the width of the rail base. For the 136RE rail, this was 5.91 inches. This gave a continuous foundation stiffness defined as an input to the model of 3,000 psi / 5.91 in, or 507.6 psi/inch.

The discretely-supported elastic foundation used in Model 2 requires partitioning the base of the rail into surfaces representing the areas where a physical rail would be in contact with a tie plate. Additionally, the concept of an effective support ratio was developed to implement this model. To calculate this ratio, it was assumed that the rail was supported by regularlyspaced ties with center-to-center spacing of 20 inches. It was further assumed that each tie plate was in contact with the underside of the rail for a length of 7.75 inches. Thus, the effective support ratio is 7.75 inches/20 inches, or 0.39 . This ratio is then used to calculate the linear elastic foundation stiffness acting over the discrete area of support. For the discretely-supported model using a 3,000 psi equivalent foundation stiffness, the continuous foundation input value of $507.6 \mathrm{psi} / \mathrm{inch}$ is then divided by this effective support ratio of 0.39. Thus, the stiffness input to a model with support only under each discrete length of rail representing a crosstie is 3,000 psi / 10

This material is declared a work of the U.S. Government and is not subject to copyright protection in the United States. Approved for public release; distribution is unlimited. 
5.91in / 0.39, or 1,309.97 psi/inch. A side view of a rail with regions of discrete support is shown in Figure 12.

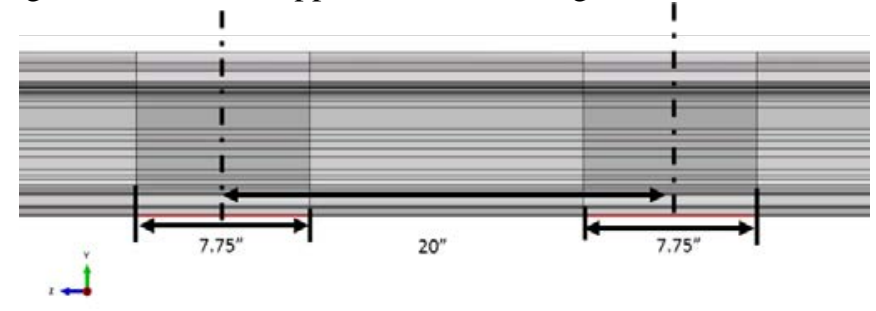

Figure 10. Illustration of Supported Distance and Tie-to-tie Spacing in Model

\section{APPENDIX B - CRACK MODELING DETAILS}

Key lessons from this examination related to how the crack tip and crack front were defined within the model. These phrases are related to how the Abaqus software defines the line corresponding to the boundary of the crack, and defines the first "ring" of elements surrounding the crack tip used to calculate $\mathrm{K}_{\mathrm{I}}$. In models Bar-1 through Bar-6, both the crack tip and crack front were defined as the same line representing the edge of where the crack bordered on the intact material within the bar. These results all exhibited an oscillation in the Contour- 1 result, which is the contour closest to the crack tip. In model Bar-7, the crack tip was defined as the same line as in the previous models, but the crack front was instead defined as the first "ring" of elements surrounding the crack tip. Figure 13 illustrates the difference between the two approaches, with the mesh hidden for clarity. The effect of this change was to eliminate the oscillation seen in Contour 1 from the Bar- 1 through Bar- 6 results. Thus, future models should use the approach from Bar-7 to define the crack front as the first ring of elements around the crack, and not the line representing the crack itself.

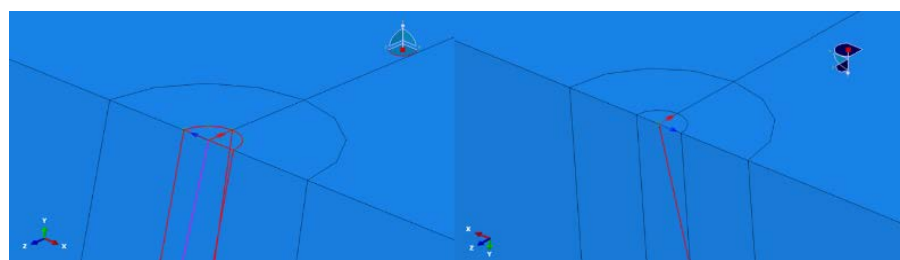

Figure 11. Crack Front and Crack Tip for Bar-7 (left) and Bar-1 through Bar-6 (right)

This material is declared a work of the U.S. Government and is not subject to copyright protection in the United States. Approved for public release; distribution is unlimited. 